\title{
Sagnac Loop in Ring Resonators for Tunable Optical Filters
}

\author{
Carmen Vázquez, Member, IEEE, Member, OSA, Salvador Elías Vargas, \\ and José Manuel Sánchez Pena, Senior Member, IEEE
}

\begin{abstract}
General filter architecture using co- and counterpropagation signals are studied. A specific configuration based on a Sagnac loop within a ring resonator is analyzed. Novel tuning, apart from conventional tuning, is achieved by changing the coupling ratio of a coupler through the adjustment of the equivalent loop length. Full equations describing the filter behavior in passive and active configurations, and simple closed-form formulas to compute the tuning, tolerance, and full-width at half-maximum are reported. The performance of these devices is discussed for their application as selective or channel-dropping ultra-narrowband filters. The effect of losses and their dispersion properties are also discussed. These devices can be conveniently implemented, using silicon- or InP-integrated optic technology, for they have high free spectral ranges.
\end{abstract}

Index Terms-Microresonators, optical filters, ring resonators, Sagnac loop, tuning and optical couplers.

\section{INTRODUCTION}

$\mathbf{T}$ UNABLE optical filters will be essential components in future reconfigurable optical networks. They should have ultra-narrow-bandwidth for use in dense-wavelength-division multiplexing (DWDM) systems with carrier spacing of $50 \mathrm{GHz}$ or less. On the other hand, the utilization of subcarriermultiplexed (SCM) data channels in DWDM optical networks has many potential important roles including packet addressing, performance monitoring using subcarriers, and network management and control. These systems require an efficient method to monitor, extract, and potentially erase subcarrier information. In this respect, optical filtering techniques have been used to simplify SCM receiver designs [1]. These filters must have high rejection ratios and free spectral ranges (FSRs) in the order of tenths of gigahertz. Narrow-band optical filters are also useful in photonic links that engage in high-speed optical RF signals. Another interesting approach is to have filter structures that can be monolithically integrated in a chip.

Common filter technology available includes arrayedwaveguide gratings, thin-film dielectric interference filters, conventional fiber Bragg gratings (FBGs), fiber Fabry-Pérot filters, and Mach-Zehnder (MZ) interferometers. A complete review, including most of these technologies, can be seen in [2]-[4]. However, these devices have difficulty providing either

Manuscript received October 14, 2004; revised April 5, 2005. This work was supported by the Spanish Ministry of Education and Science CICYT (TIC200303783).

The authors are with the Electronic Technology Department, University Carlos III of Madrid, 28911 Madrid, Spain (e-mail: cvazquez@ing.uc3m.es).

Digital Object Identifier 10.1109/JLT.2005.850793 notch or bandpass filters with ultranarrow bandwidths. These requirements can be covered by optical filters based on ring resonators (RRs) and loop mirrors [4]-[12]. These ring-based filters have been utilized in numerous linear and nonlinear optical applications [10], including biochemical sensors [14] and all-optical switches [13]. Even various compound RRs [15]-[17] and discontinuity-assisted RRs [18], which are constructed by an RR and a reflective section within it, are reported and analyzed by different methods. However, these filters are tuned by varying the phase delay of the waveguides, and the reflective section is implemented either by a Fabry-Pérot cavity, by a grating, or by a waveguide with a different refractive index.

Tunable filters based on RR and a Michelson interferometer (MI) as the reflective element in the feedback path are reported in [19], with the MI made of a directional coupler and two identical FBGs. But in this MI configuration, the fabrication tolerances impose a limit in the balance of the interference paths, and consequently, in the filter behavior. The use of a Sagnac interferometer as the reflective element [20] alleviates this problem, because of its common-path architecture. This configuration is experimentally tested in its passive form in [21]. In any of these configurations, it is necessary to have a variable coupling coefficient, and its accuracy and tolerances must be analyzed. Tolerance effects on an RR with a FBG filter in transmission mode within it are reported in [7], where it was also proposed that it be controlled by using a variable loss or gain. Coupling coefficient tolerance effect, in terms of tuning capabilities, has not yet been analyzed.

In this paper, we report a detailed study of the general architecture of a tunable filter, based on an RR and a reflective section in the feedback path, working in its passive and active forms, depending on the application. The filter response is calculated using two different methods, the transfer matrix formalism and the $z$-transform technique, and the same expressions are obtained independently. Losses are included in the model. Simple equations are shown for helping the design process when using a specific reflective section, a Sagnac loop. The present analysis is restricted to couplers characterized by two parameters, the power coupling coefficient, and the power excess loss. Tuning is achieved by changing the phase delay in the ring or Sagnac waveguides and additionally by changing the coupling coefficient of the Sagnac loop. Simple closed-form formulas describing the novel tuning process are derived in its active and passive configurations, along with the requirements for having a certain operation as a bandpass 
or bandstop filter. Measurements on a fiber-optic prototype validating the theoretical model, and the tunability, are reported elsewhere [21]. Coupling coefficient tolerance effect, in terms of tuning capabilities, is also analyzed. These filters can be implemented in optical fiber technology and in silicon- or InPintegrated optic technologies, because photonic circuits with equivalent components have already been developed. Some of them are a monolithically integrated Sagnac interferometer for an all-optical controlled-NOT gate [22], filters using active RRs [23], [24], passive single- and double-RRs [8], [25]-[27] and microcavities [28]. The resonant frequencies of the proposed device can be shifted by changing the equivalent loop length by carrier injection [29] or local heating [30], as in any RR-based device. The transfer function can be tailored by changing the loop loss; and if III-V materials are used in the fabrication, this can be done by electroabsorption [31]. The filter resonant frequencies can also be changed by tuning the coupling ratio, which up to now is technologically possible by adjusting the taper-resonator gap fabricated by stretching a standard optical fiber [28]; by a micromechanical-fiber variable-ratio coupler, using microelectromechanical systems (MEMS) actuated deformable waveguides [32], or by using electrical control of waveguide-resonator coupling in an MZ coupler configuration [33], [34]. Although present analysis is particularized to couplers characterized by two parameters, general equations can be particularized to each technological solution.

The paper is focused on the presentation of the architecture, and it is structured as follows. Section II presents the general architecture and its basic theoretical description, including the transfer function for the different output ports. In Section III, a specific scheme using a Sagnac loop is described in its active and passive forms, and tuning equations are reported. Section IV elaborates on different filter applications, closedform expressions for calculating some relevant design parameters, coupling-ratio tolerances, and the filter dispersion properties. Finally, Section V elaborates on the summary and conclusion.

\section{Filter Architecture And General Equations}

In this section, we present the general architecture of the proposed filter, which consists of compound devices in a ring configuration with a reflective section. From a digital filter point of view, they are infinite impulse response (IIR) filters whose poles can be located radially using a gain or loss and axially using a coupling coefficient $K$. For a filter made up of a single stage, this property implies that tuning can be achieved through the $K$ value. The transfer function of the filter is derived using two methods: the general matrix formalism, as in [16], and the $z$-transform technique [4].

The filter architecture is shown in Fig. 1. It is made up of an RR, a transmission-reflection function (TRF), which represents the reflective section, and different transmission functions before and after the TRF. This TRF has a transfer function in reflection and in transmission named FR and FT, respectively.

\section{A. General Equations Using the Matrix Formalism}

The transfer-matrix method is used for calculating the transfer function of a compound device that is made of concatenated elementary devices; and each of them can be represented by its own transfer matrix. In our filter, there are clockwise and counterclockwise propagation, so the device is unfolded into two simple traveling-wave resonators connected by the TRF (Fig. 2), as reported in [16], where a compound-fiber RR is also analyzed. Afterwards, the transmission matrix of the coupler, the waveguides, and the TRF will be used.

The transfer function of the filter can be straightforwardly obtained by applying

$$
\left[\begin{array}{c}
E_{1} \\
E_{3}
\end{array}\right]=[a c][g][\mathrm{TRF}]\left[g^{\prime}\right]\left[a c^{\prime}\right]\left[\begin{array}{c}
E_{1}^{\prime r} \\
E_{3}^{\prime}
\end{array}\right]
$$

where $E_{1}^{\prime r}$ is the reflected output at port $E_{1}, E_{3}^{\prime}$ is the input power at port $E_{3}$ (which in our analysis is equal to 0 ), while $E_{1}$ is the input and $E_{3}$ is the output (see Fig. 2). The expressions of the matrices of (1) can be seen in Appendix A.

From the above expressions, it can be derived that the output transfer function at port $E_{3}$ is given by (2), shown at the bottom of page, where $K_{1}$ and $\gamma_{1}$ are the coupling coefficient and the excess loss of the input coupler, respectively. $\alpha$ and $\beta$ are the waveguide attenuation and the propagation constant, respectively, $L_{\mathrm{T}}$ is the total waveguide length, and $l_{a}$ is the waveguide length connecting the input coupler and the TRF (see Fig. 1), $\Pi_{1}^{N}\left(T_{x_{i}}\right)$ and $\Pi_{1}^{M}\left(T_{y_{i}}\right)$ are the $N$ transmission transfer functions between the input coupler and the TRF, and the $M$ transmission transfer functions between the TRF and the input coupler, respectively.

From (2), it is observed that the TRF position within the RR has no importance, because there is no dependence on $l_{a}$. But it is important in deriving the reflected output transfer function at port $E_{1}$, which is given by (3), shown at the bottom of the next page.

$$
\begin{aligned}
& \frac{E_{3}}{E_{1}}=\left(1-\gamma_{1}\right)^{\frac{1}{2}}
\end{aligned}
$$

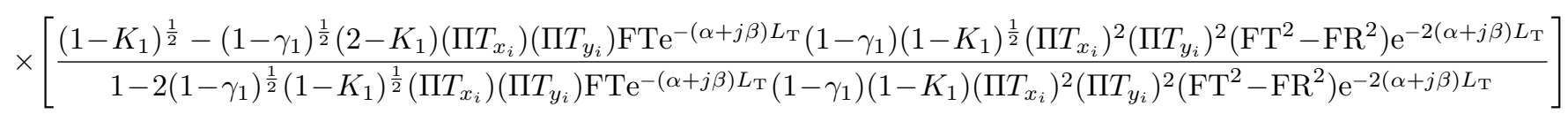




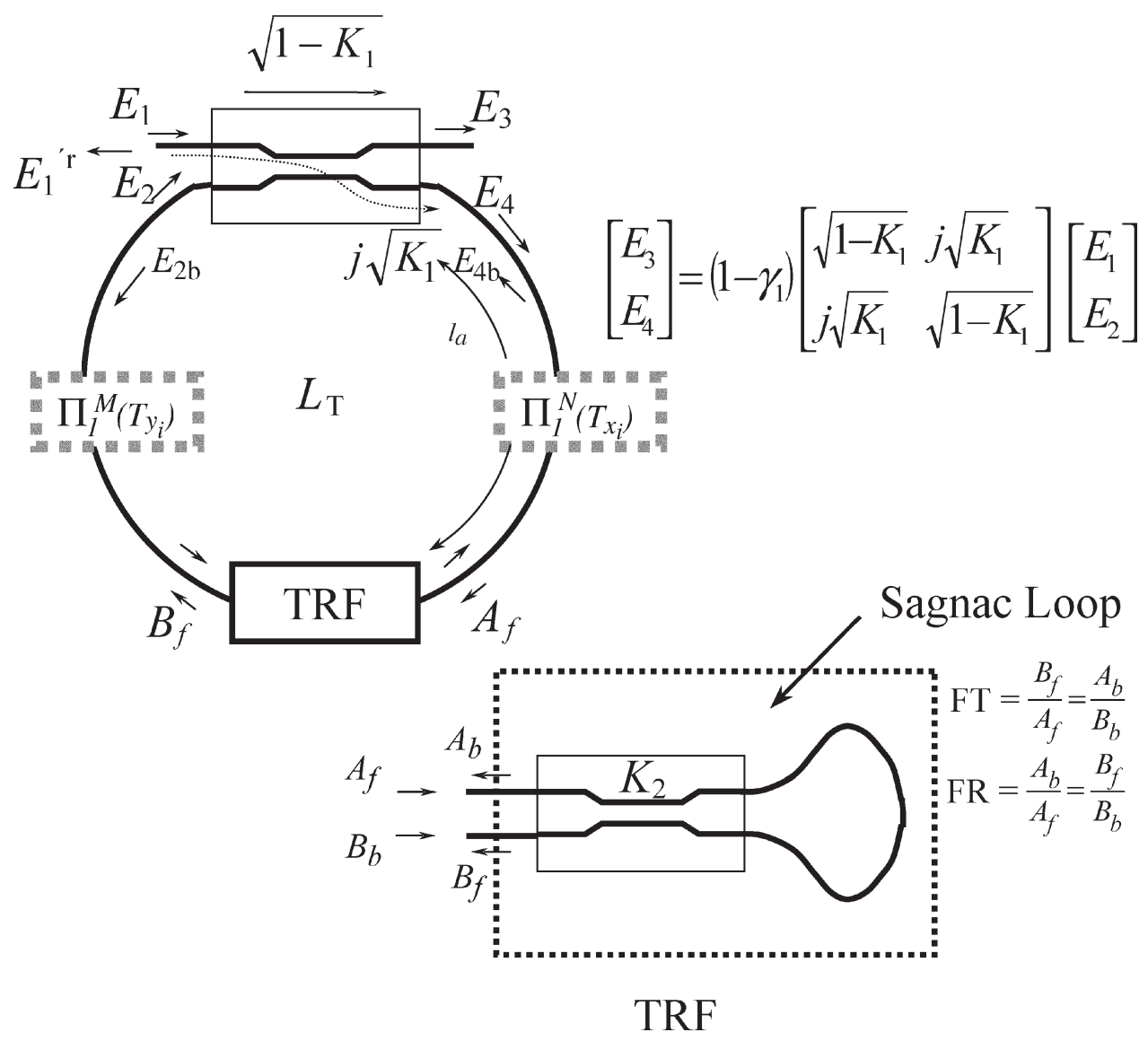

Fig. 1. General filter architecture. $T_{x_{i}}$ and $T_{y_{i}}$ are any of the $N$ and $M$ transmission transfer functions placed before and after the TRF, and $L_{\mathrm{T}}$ is the ring total length. The inset is a schematic of a loop Sagnac as the TRF.

Both transfer functions [see (2) and (3)] have the same denominator, which can be factorized as

$$
\begin{aligned}
F_{1} \times F_{2}=\left[1-\left(1-\gamma_{1}\right)^{\frac{1}{2}}\left(1-K_{1}\right)^{\frac{1}{2}}\left(\Pi T_{x_{i}}\right)\left(\Pi T_{y_{i}}\right)\right. \\
\left.\times(\mathrm{FT}+\mathrm{FR}) \mathrm{e}^{-(\alpha+j \beta) L_{\mathrm{T}}}\right] \\
\times\left[1-\left(1-\gamma_{1}\right)^{\frac{1}{2}}\left(1-K_{1}\right)^{\frac{1}{2}}\left(\Pi T_{x_{i}}\right)\left(\Pi T_{y_{i}}\right)\right. \\
\left.\times(\mathrm{FT}-\mathrm{FR}) \mathrm{e}^{-(\alpha+j \beta) L_{\mathrm{T}}}\right]
\end{aligned}
$$

There is only one difference in these two factors, the term related to FT and FR, in the first factor $F_{1}$ they are added up, and in the second one $F_{2}$ they are subtracted.

The $\lambda$ values, which make any of these two factors 0 , are the resonance wavelengths. Selecting FT and FR in a way that FR is an imaginary number and FT is a real number (or

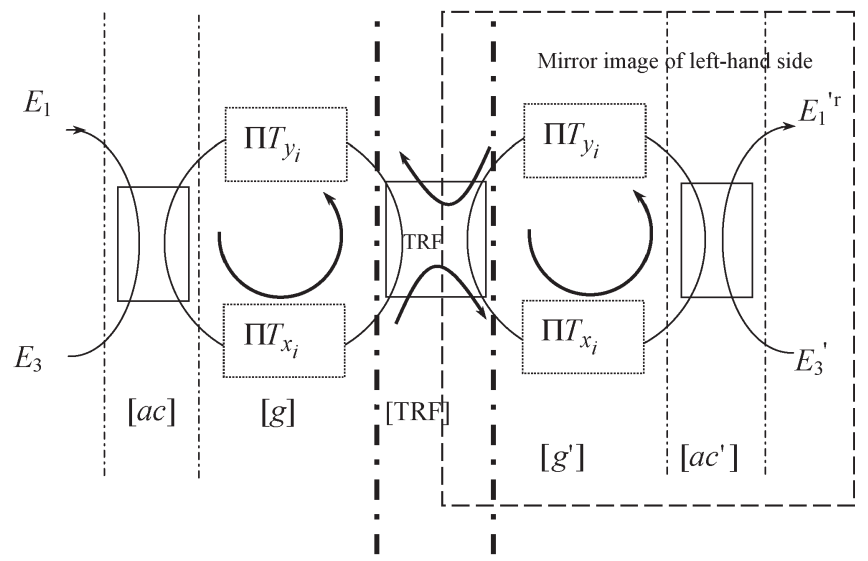

Fig. 2. Unfolded equivalent model of the compound ring resonator.

vice versa), we can have phase dependence for controlling the resonance wavelengths location. A specific example is developed in Section III.

$$
\frac{E_{1}^{\prime r}}{E_{1}}=\frac{-\left(1-\gamma_{1}\right) K_{1}\left(\Pi T_{x_{i}}\right)^{2} \mathrm{FRe}^{-2(\alpha+j \beta) l_{a}}}{1-2\left(1-\gamma_{1}\right)^{\frac{1}{2}}\left(1-K_{1}\right)^{\frac{1}{2}}\left(\Pi T_{x_{i}}\right)\left(\Pi T_{y_{i}}\right) \mathrm{FTe}^{-(\alpha+j \beta) L_{\mathrm{T}}}\left(1-\gamma_{1}\right)\left(1-K_{1}\right)\left(\Pi T_{x_{i}}\right)^{2}\left(\Pi T_{y_{i}}\right)^{2}\left(\mathrm{FT}^{2}-\mathrm{FR}^{2}\right) \mathrm{e}^{-2(\alpha+j \beta) L_{\mathrm{T}}}}
$$




\section{B. General Equations Using the z-Transform Technique}

Another approach for obtaining the compound-device transfer function has been used, the $z$-transform technique. This is commonly used in designing photonic filters from a digital perspective [4]. A second-order polynomial in the denominator is obtained, so transfer functions not available with a single $\mathrm{RR}$ can be achieved. Our main purposes are to corroborate the expressions derived in Section I-A and obtaining the pole and zero locations for synthesis purposes, which will be described elsewhere.

In order to be able to correctly apply the $z$-transform technique, the IIR of the device must have a unitary delay $(\tau)$. The signal that passes through the TRF travels a total length $L_{\mathrm{T}}$, and the reflected signal at the TRF travels twice this total length, so $\tau=n_{\mathrm{ef}} L_{\mathrm{T}} / c$, where $n_{\mathrm{ef}}$ is the waveguide effective refractive index, and $c$ is the light speed in a vacuum.

The following transfer functions at port $E_{3}$ are obtained (see Appendix B):

$$
\frac{E_{3}}{E_{1}}=\left(1-\gamma_{1}\right)^{\frac{1}{2}}\left(1-K_{1}\right)^{\frac{1}{2}}\left[\frac{1+B z^{-1}+C z^{-2}}{\left(1-Z p_{1} z^{-1}\right)\left(1-Z p_{2} z^{-1}\right)}\right]
$$

where $Z_{p_{1}}$ and $Z_{p_{2}}$ are the poles of the transfer function and are given by

$$
\begin{aligned}
& Z p_{1}=\left(1-\gamma_{1}\right)^{\frac{1}{2}}\left(1-K_{1}\right)^{\frac{1}{2}} \prod_{i=1}^{N} T_{x_{i}} \prod_{i=1}^{M} T_{y_{i}}[\mathrm{FT}+\mathrm{FR}] \\
& Z p_{2}=\left(1-\gamma_{1}\right)^{\frac{1}{2}}\left(1-K_{1}\right)^{\frac{1}{2}} \prod_{i=1}^{N} T_{x_{i}} \prod_{i=1}^{M} T_{y_{i}}[\mathrm{FT}-\mathrm{FR}]
\end{aligned}
$$

where FR and FT are, respectively, the transfer function in reflection and in transmission of the TRF, and $z^{-1}$ represents the unit delay related to $L_{\mathrm{T}}$.

$B$ and $C$ are given by

$$
\begin{aligned}
& B=\frac{\left(1-\gamma_{1}\right)^{\frac{1}{2}}\left(K_{1}-2\right) \prod_{i=1}^{N} T_{x_{i}} \cdot \prod_{i=1}^{M} T_{y_{i}} \cdot \mathrm{FT}}{\left(1-K_{1}\right)^{\frac{1}{2}}} \\
& C=\left(1-\gamma_{1}\right) \prod_{i=1}^{N} T_{x_{i}} \prod_{i=1}^{M} T_{y_{i}}\left[\mathrm{FT}^{2}-\mathrm{FR}^{2}\right] .
\end{aligned}
$$

This new representation of the transfer function can be used to determine the value range of the device parameters for designing a stable filter. The modulus of the poles [from (6) and (7)] must be within the unit circle to avoid an infinite transmission, i.e., laser oscillation.

The transfer function at port $E_{3}$, given by (2) in Section II-A, and by (5) in this section, are equal, and the two factors of the denominator given by (3) are the two poles described in (6) and (7). This is a way of verifying that all the expressions are correctly derived.

\section{Ring Resonator With a SAgnac Loop}

In this section, a specific tunable filter design is proposed The design includes an active RR, and the TRF in the RR is implemented by a Sagnac Loop. A schematic of the proposed filter is shown in Fig. 1, using the TRF described in the inset. The amplifier, with a power gain $G$, amplifies the clockwise and the counterclockwise signals, so no isolators are considered.

The Sagnac interferometer has been used for extracting modulated channels in a subcarrier in WDM networks [1], as a two-position switch with FBGs [35], with an active RR as a laser of two ultrafine lines [36], and with an RR as a birefringence magnifier [37], apart from numerous sensor applications.

In this design, it is used as a mirror with variable reflectance, generating variable clockwise and counterclockwise signals that interfere with each other. In comparison with other alternatives, such as an MI [19], it is easier to physically implement working in a coherent regimen, because the two arms of the interferometer are of the same fiber, making the alignment straightforward. In the case of two physically different arms, i.e., made of theoretically two identical FBGs, the tolerance in fabrication could alter the desired equal length between both paths.

The Sagnac interferometer is a device having a TRF that partially transmits (FT) and reflects (FR), the input light. These functions are given by

$$
\begin{aligned}
& \mathrm{FR}=j 2\left(1-\gamma_{2}\right) \sqrt{K_{2}\left(1-K_{2}\right)} \\
& \mathrm{FT}=\left(1-\gamma_{2}\right)\left(1-2 K_{2}\right)
\end{aligned}
$$

where $\gamma_{2}$ and $K_{2}$ are the excess loss and the coupling coefficient of the coupler connecting the Sagnac loop, respectively (see inset of Fig. 1). In (10) and (11), waveguide attenuation and retardation in signal propagation are not included, because both have already been considered in general in (2) and (3).

In the following, the frequencies at which transfer functions will have a maximum or a minimum and any condition required to effectively have them are going to be determined. A description of how these frequencies can be tuned is also reported.

\section{A. Maximum Resonance Condition and Frequency Location}

The maximum frequencies of the transfer function can be derived by requiring both the real and the imaginary components of the denominator of $E_{3}$ to be equal to 0 . This denominator, for the specific design with a Sagnac Loop, is obtained by substituting (10) and (11) in (3). The two factors $F_{1}$ and $F_{2}$ are written as

$$
\begin{aligned}
& F_{1}=\left[1-\left(1-\gamma_{1}\right)^{\frac{1}{2}}\left(1-K_{1}\right)^{\frac{1}{2}} G^{\frac{1}{2}}(\mathrm{FT}+\mathrm{FR}) \mathrm{e}^{-(\alpha+j \beta) L_{\mathrm{T}}}\right] \\
& F_{2}=\left[1-\left(1-\gamma_{1}\right)^{\frac{1}{2}}\left(1-K_{1}\right)^{\frac{1}{2}} G^{\frac{1}{2}}(\mathrm{FT}-\mathrm{FR}) \mathrm{e}^{-(\alpha+j \beta) L_{\mathrm{T}}}\right]
\end{aligned}
$$


where

$$
\begin{aligned}
& \mathrm{FT}+\mathrm{FR}=\left(1-\gamma_{2}\right) \mathrm{e}^{j \phi_{\mathrm{s}}} \\
& \mathrm{FT}-\mathrm{FR}=\left(1-\gamma_{2}\right) \mathrm{e}^{-j \phi_{\mathrm{s}}}
\end{aligned}
$$

and

$$
\phi_{\mathrm{s}}= \pm \arg \left(\frac{2 \sqrt{K_{2}\left(1-K_{2}\right)}}{\left(1-2 K_{2}\right)}\right) .
$$

Both factors $F_{1}$ and $F_{2}$ are 0 if

$$
G^{\frac{1}{2}}=\frac{1}{\left(1-\gamma_{1}\right)^{\frac{1}{2}}\left(1-\gamma_{2}\right)\left(1-K_{1}\right)^{\frac{1}{2}} \mathrm{e}^{-\alpha L_{\mathrm{T}}}}=G p^{\frac{1}{2}}
$$

and

$$
2 \pi k=\frac{2 \pi f_{1,2} n_{\mathrm{ef}}}{c} L_{\mathrm{T}} \mp \phi_{\mathrm{s}}, \quad k=1,2,3, \ldots
$$

where $f_{1,2}$ is the frequency in hertz and the subscripts " 1 " and " 2 " refer to the factors $F_{1}$ and $F_{2}$, respectively.

$G_{\mathrm{p}}$ is a new parameter that we have defined, it is named pole gain, and it represents the resonance condition of the maxima.

From (18), we obtain the FSR of the compound device as

$$
\mathrm{FSR}=\frac{c}{n_{\mathrm{ef}} L_{\mathrm{T}}}
$$

The maximum frequencies are derived from (18), and the amplitude of the transfer function at these frequencies (the resonance peak) can be made arbitrarily narrow and sharp. When $G_{\mathrm{p}}=1$, we obtain, according to (2), an infinite transmission, i.e., laser oscillation. $G_{\mathrm{p}}$ can be used as a parameter to control the peak resonance value.

\section{B. Maximum Frequencies Tuning by Changing the Coupling Coefficient}

The poles of the transfer function are complex conjugated numbers, which are obtained by substituting (14) and (15) into (6) and (7), and their phases depend not only on the optical path, but also on $K_{2}$. This phase determines the maximum frequency, which consequently, can be controlled through the coupling coefficient. The same conclusion can be derived from (16) and (18). If we want to tune the filter maximum of order $k$ to a specific frequency $f_{0}$, we first obtain

$$
k=\frac{f_{0}}{\mathrm{FSR}} .
$$

From (18) and (19), we obtain the maximum frequency as

$$
f_{1,2}=f_{0} \pm \frac{c \phi_{\mathrm{s}}}{2 \pi n_{\mathrm{ef}} L_{\mathrm{T}}} .
$$

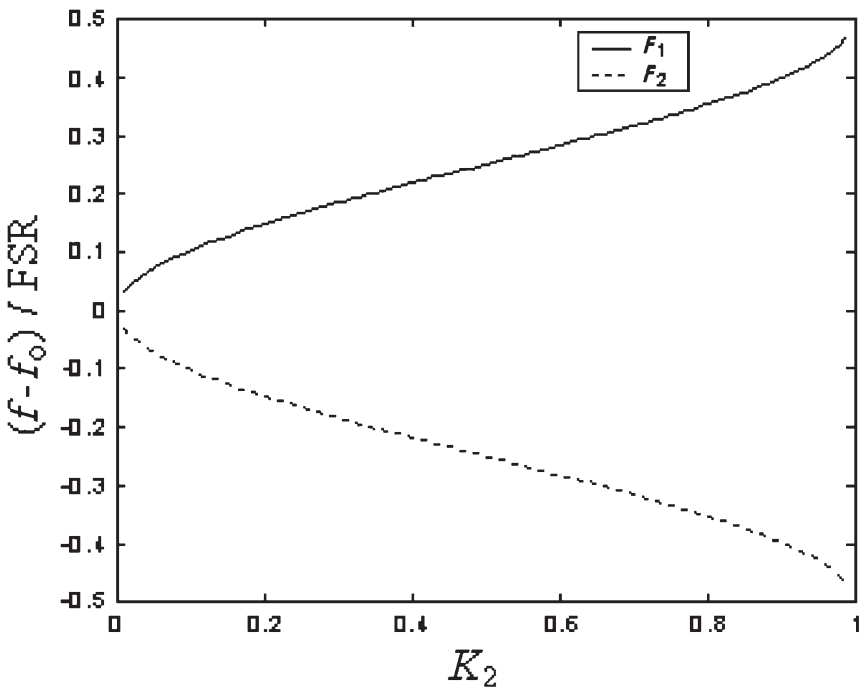

Fig. 3. Normalized maximum frequency $\left(f_{0}\right)$ separation $\left(f-f_{0}\right) / \mathrm{FSR}$, of an RR with a Sagnac loop, versus the coupling coefficient $\left(K_{2}\right)$.

From (21), it is shown that for a certain $K_{2}$ value, there are two maximum frequencies that are symmetric with respect to $f_{0}$, as can be seen in Fig. 3. It is remarked which factor, $F_{1}$ or $F_{2}$, is the one responsible for every maximum frequency.

On the other hand, by changing $\phi_{\mathrm{s}}$ with $K_{2}$, the maximum frequency is tuned and the greatest variation becomes equal to FSR/2. A specific example of an RR having an FSR of $19.224 \mathrm{GHz}, K_{1}=0.1, \gamma_{1}=\gamma_{2}=0.05$, and $G=1.28$, and different $K_{2}$ values can be seen in Fig. 4. The location of the maximum frequencies is obtained with two different methods: the first one simulates the transfer function with (5) or (2), and the second one uses the explicit expression (21), and the same results are obtained.

\section{Minimum Resonance Condition, Frequencies Location, and Tuning}

The minimum frequencies of the transfer function can be derived by requiring both the real and imaginary components of the numerator of $E_{3}$ to be equal to 0 . This numerator is factorized in two terms $F_{c 1}$ and $F_{c 2}$ given by

$$
\begin{aligned}
F_{c 1}= & 1-\frac{\left(\left(1-\gamma_{1}\right) G\right)^{\frac{1}{2}}}{2\left(1-K_{1}\right)^{\frac{1}{2}}} \\
& \times\left[\left(2-K_{1}\right) \mathrm{FT}+\sqrt{K_{1}^{2} \mathrm{FT}^{2}+4 \mathrm{FR}^{2}\left(1-K_{1}\right)}\right] \\
& \times \mathrm{e}^{-(\alpha+j \beta) L_{\mathrm{T}}} \\
F_{c 2}= & 1-\frac{\left(\left(1-\gamma_{1}\right) G\right)^{\frac{1}{2}}}{2\left(1-K_{1}\right)^{\frac{1}{2}}} \\
& \times\left[\left(2-K_{1}\right) \mathrm{FT}-\sqrt{K_{1}^{2} \mathrm{FT}^{2}+4 \mathrm{FR}^{2}\left(1-K_{1}\right)}\right] \\
& \times \mathrm{e}^{-(\alpha+j \beta) L_{\mathrm{T}}} .
\end{aligned}
$$


(a)

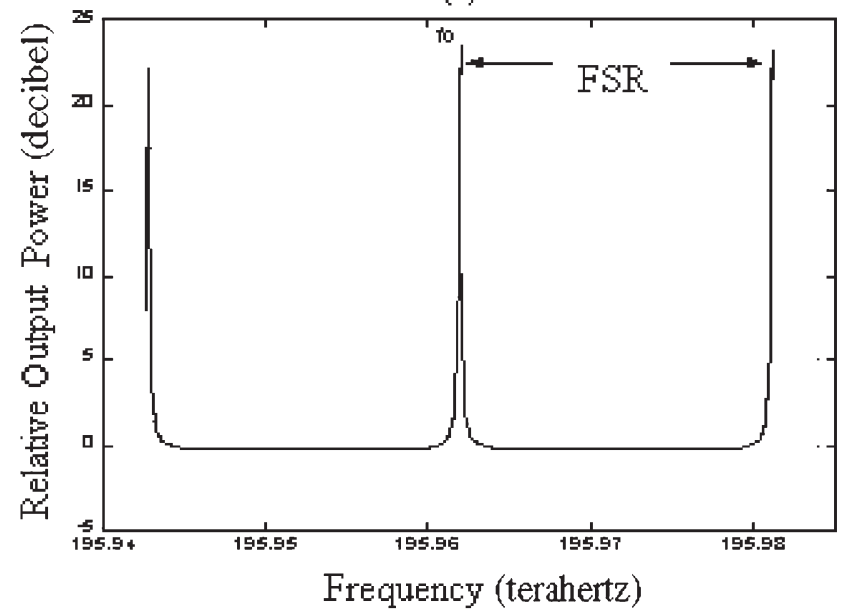

(c)

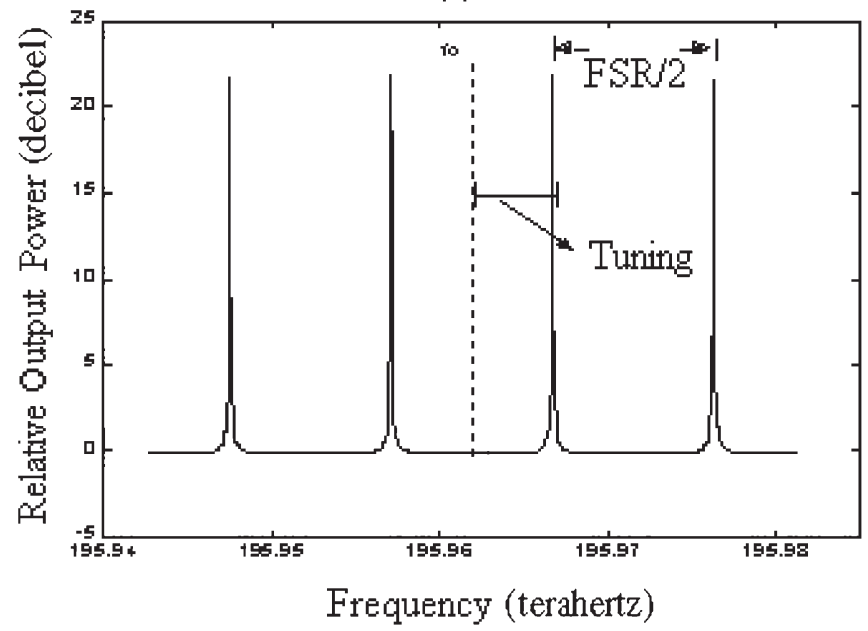

(b)

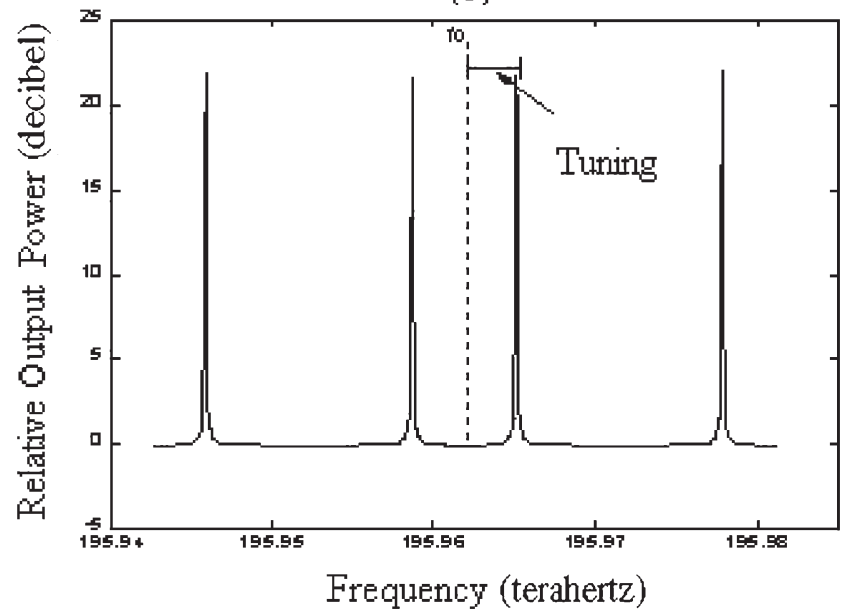

(d)

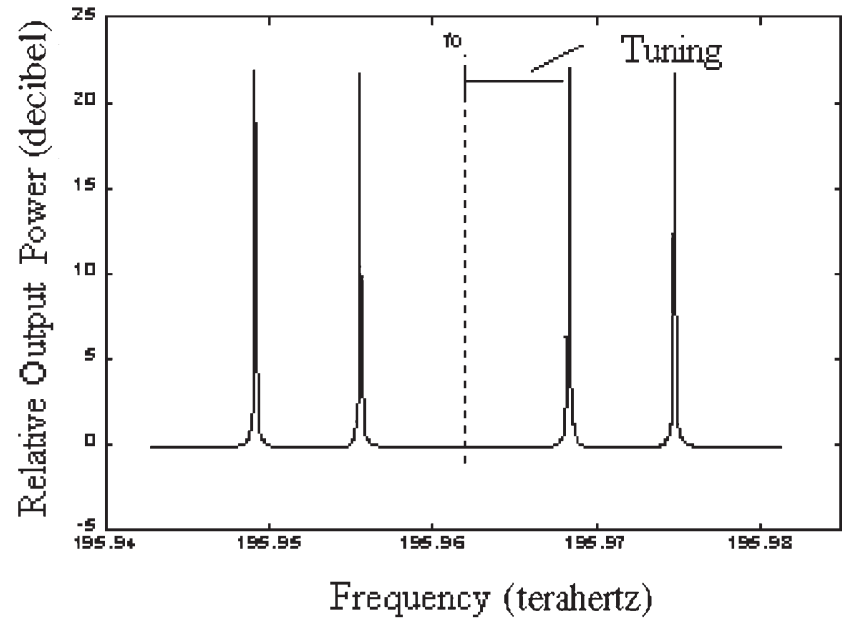

(e)

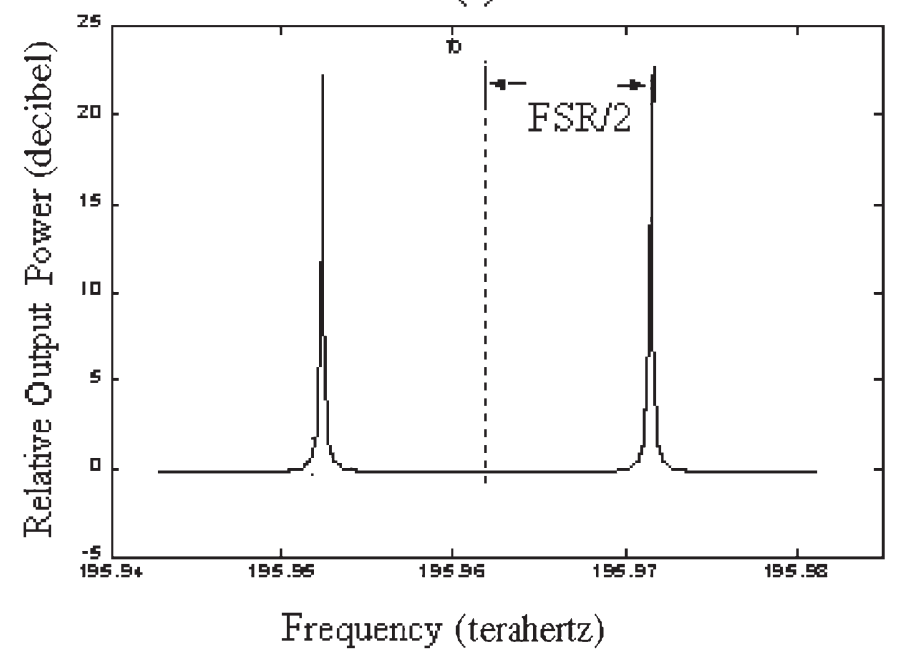

Fig. 4. Tuning of the transfer function of the active RR with a Sagnac loop: $K_{1}=0.1, \gamma_{1}=\gamma_{2}=0.05, G=1.28$, and for $K_{2}$ values of (a) 0 , (b) 0.25 , (c) 0.50 , (d) 0.75 , or (e) 1 .

At port $E_{1}^{\prime r}$, the numerator is a polynomial of first order with a constant phase, so there are no tuning zero frequencies.

The second term of (22) has a radical that is equal in both factors and can be positive or negative. Our aim is to obtain a phase dependence different to the optical path, so this second term must be a complex number. Substituting FR and FT with (10) and (11), respectively, the radical is negative if

$$
K_{1}<-8 A+4 \sqrt{4 A^{2}+A}
$$


where

$$
A=\frac{K_{2}\left(1-K_{2}\right)}{\left(1-2 K_{2}\right)^{2}} .
$$

For $K_{2}$ values close to 0.5 , it is greater the range of $K_{1}$ values that gives complex numbers. Substituting $K_{2}$ with $1-$ $K_{2}$ in (25), the same $A$ value is obtained.

Assuming the designed device follows (24) and (25), the factors $F_{c 1}$ and $F_{c 2}$ are 0 if

$$
G^{\frac{1}{2}}=\frac{1}{\left(1-\gamma_{1}\right)^{\frac{1}{2}}\left(1-\gamma_{2}\right) \mathrm{e}^{-\alpha L_{\mathrm{T}}}}=G_{\mathrm{c}}^{\frac{1}{2}}=G_{\mathrm{p}}^{\frac{1}{2}} \sqrt{1-K_{1}}
$$

and

$$
2 \pi m=\frac{2 \pi f_{z 1,2} n_{\mathrm{ef}}}{c} L_{\mathrm{T}} \mp \phi_{z}, \quad m=1,2,3, \ldots
$$

where

$$
\begin{aligned}
\phi_{z} & =\phi_{z 1}=-\phi_{z 2} \\
& =\arg \left(\frac{\sqrt{-K_{1}^{2}\left(1-2 K_{2}\right)^{2}+16\left(1-K_{1}\right)\left(1-K_{2}\right) K_{2}}}{\left(2-K_{1}\right)\left(1-2 K_{2}\right)}\right) .
\end{aligned}
$$

In these expressions, $\phi_{z 1}, \phi_{z 2}$ are the phases and $f_{z 1}, f_{z 2}$ are the minimum frequencies (the "1" and "2" subscripts refer to the factor $F_{c 1}$ and $F_{c 2}$, respectively).

$G_{\mathrm{C}}$ is a new parameter that we define, which represents the minimum resonance condition and is named the zero gain. The condition shown in (26), known in the microwave field as critical coupling, is due to a perfect destructive interference.

From (27) and (28), we see that tuning of the minimum frequencies is controlled through $K_{1}$ and $K_{2}$. A wider tuning range is obtained for $K_{2}$ close to 1 , with $K_{1}$ small, and a worse tuning is achieved for $K_{1}$ close to 0 and $K_{2}$ close to 0 , or if $K_{1}$ is close to 1 and $K_{2}$ approaches 0.5 (from $K_{2}<0.5$ ). As a general rule, we select a small and fixed $K_{1}$ value, and we use $K_{2}$ for tuning the device. Each minimum can be tuned up to $\mathrm{FSR} / 2$.

An example of the tuning process with $K_{2}$ is shown in Fig. 5 for a $G$ value close to the $G_{\mathrm{c}}$ condition in (26): $K_{1}=0.4$, $\mathrm{FSR}=19.224 \mathrm{GHz}, \gamma_{1}=\gamma_{2}=0.05$, and $G=1.13 . K_{1}$ and $K_{2}$ values follow those of (24).

\section{Filter ApPlicAtions AND DEVICE PARAMETERS}

The active RR with the Sagnac loop can have different filter applications depending on device parameters, such as gain, coupling coefficients, and losses. Because the transfer function at port $E_{3}$ have poles and zeros, or frequencies at which the amplitude goes ideally to 0 or infinity, we can have channeldropping or selective filters at that port. The resonance conditions are different in each case, in the phase and in the amplitude conditions, so they will be used to discern between them.

The resonance conditions for the transfer function at port 3 are given by the pole and zero gains, which are plotted versus the $K_{1}$ and $G$ values, with $K_{2}$ as a parameter, at Fig. 6. It can be seen that $G_{\mathrm{p}}$ is always greater than any $G_{\mathrm{c}}$ value, as it can also be derived from (26). $G_{\mathrm{p}}$ only depends on the $K_{1}$ value. $G_{\mathrm{c}}$ is independent of $K_{1}$ and $K_{2}$ if (24) is fulfilled. In this case, the device has two complex conjugated zeros or two minimum frequencies that are symmetric with respect to the minimum frequency of the device with no reflections $\left(K_{2}=0\right)$, and there is a unique resonance condition for both minimum frequencies $G_{\mathrm{c}}$. A specific example is reported in Fig. 5, for $K_{1}=0.4$, which corresponds to the straight line shown in Fig. 6, for any $K_{2}$ value. But if (24) is not followed, there are different resonance conditions depending on the $F_{c 1,2}$ factor, which is 0 . This corresponds in Fig. 6 with the divergence in the $G_{\mathrm{c}}$ curve at a certain point, so $G_{c 1,2}$ are obtained; for example, at $K_{1}=0.6$ for $K_{2}=0.05$. The $G_{c 1,2}$ curves for any $K_{2}$ value are equal for the corresponding $1-K_{2}$ value, because the same $A$ value is obtained for any of them in (25).

In any case, the filter is stable if the transfer function poles are located inside the unit circle; from (6), (14), and (26), it is derived that this condition is fulfilled if $G<G_{\mathrm{p}}$.

The output power is obtained by substituting (10) and (11) in either (2) or (5). In the particular case of selecting $K_{1}$ and $K_{2}$ values following (24) and after some simplifications, the expression is of (29), shown at the bottom of the page, with

$$
\begin{aligned}
A_{0}|C| & =\sqrt{\left(1-\gamma_{1}\right)}\left(1-\gamma_{2}\right) \sqrt{G} e^{-\alpha L_{\mathrm{T}}} \\
& =\sqrt{\frac{G}{G_{\mathrm{c}}}} \\
D_{0} & =\sqrt{\left(1-\gamma_{1}\right)\left(1-K_{1}\right)}\left(1-\gamma_{2}\right) \sqrt{G} e^{-\alpha L_{\mathrm{T}}} \\
& =\sqrt{\frac{G}{G_{\mathrm{p}}}} .
\end{aligned}
$$

\section{A. Designing a Selective Filter}

In order to have a selective filter with a sharp response and tunable through the coupling coefficient $K_{2}$, the values of the parameters $K_{1}$ and $G$ must be those that make the ratio $G_{\mathrm{p}} / G$ close to 1 but still produce a stable filter, which means the gain must always be lower than the pole gain. An

$$
\left|\frac{E_{3}}{E_{1}}\right|^{2}=\frac{\left(1+A_{0}^{2}|C|^{2}\right)^{2}\left(1-2 \frac{A_{0}|C|}{1+A_{0}^{2}|C|^{2}} \cos \left(-\beta L_{\mathrm{T}}+\phi_{Z}\right)\right)\left(1-2 \frac{A_{0}|C|}{1+A_{0}^{2}|C|^{2}} \cos \left(-\beta L_{\mathrm{T}}-\phi_{Z}\right)\right)}{\left(1+D_{0}^{2}\right)^{2}\left(1-2 \frac{D_{0}}{1+D_{0}^{2}} \cos \left(-\beta L_{\mathrm{T}}+\phi_{\mathrm{s}}\right)\right)\left(1-2 \frac{D_{0}}{1+D_{0}^{2}} \cos \left(-\beta L_{\mathrm{T}}-\phi_{\mathrm{s}}\right)\right)}
$$


(a)

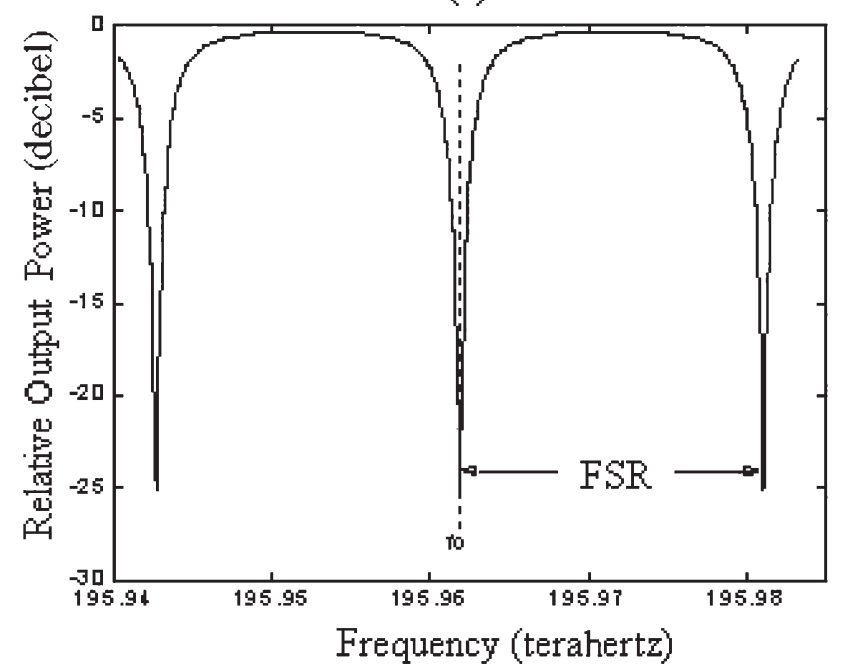

(c)

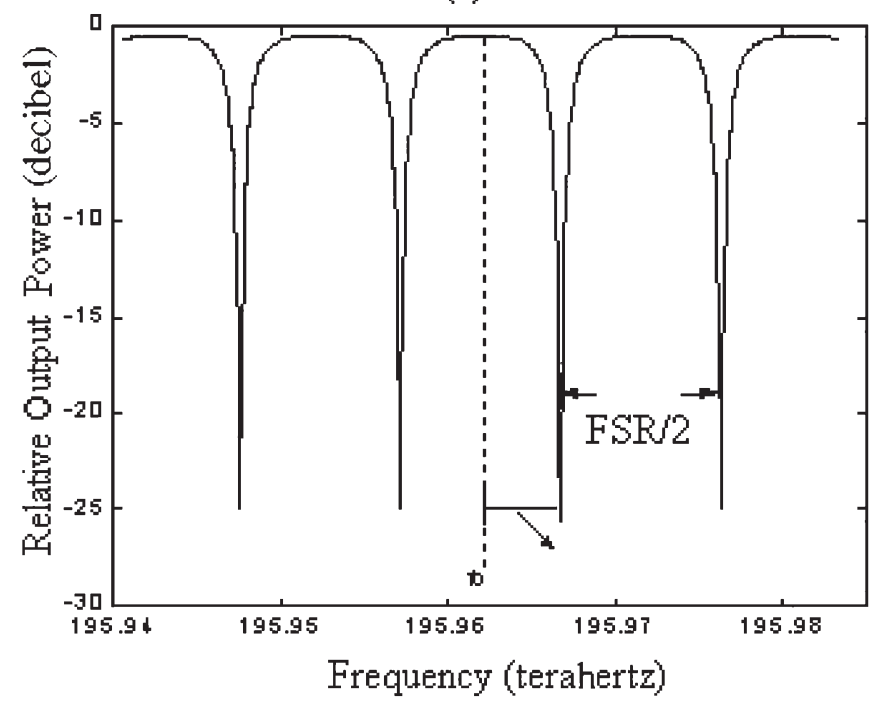

(b)

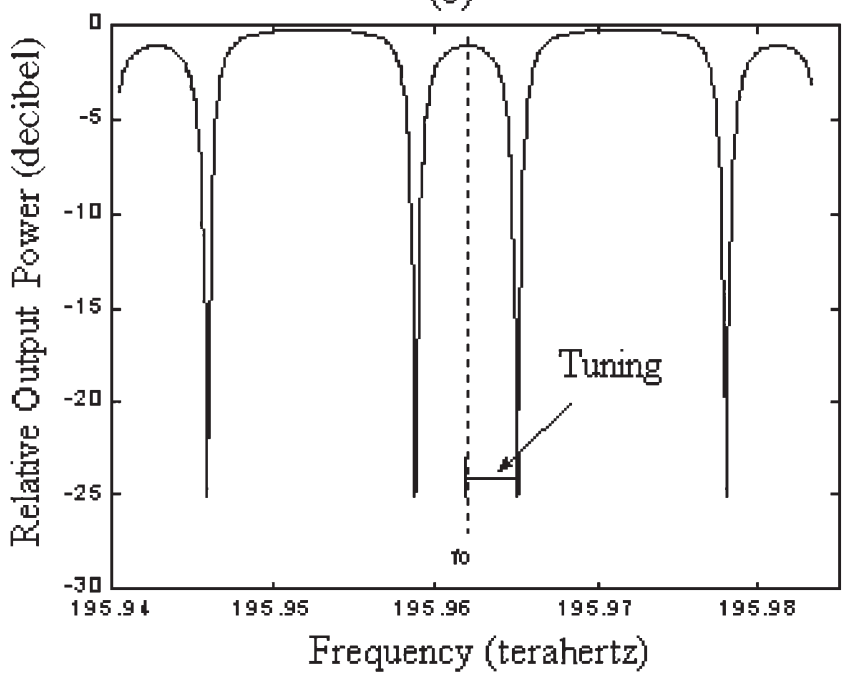

(d)

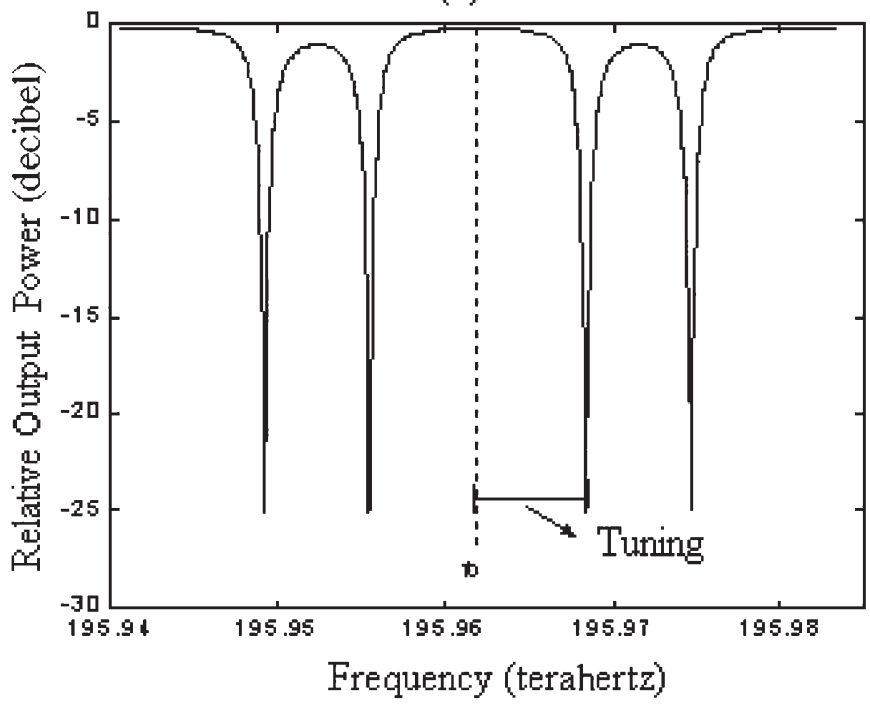

Fig. 5. Tuning of the transfer function of the active RR with a Sagnac loop: $K_{1}=0.4, \gamma_{1}=\gamma_{2}=0.05, G=1.13$, and for $K_{2}$ values of (a) 0 , (b) 0.25 , (c) 0.50 , or (d) 0.75

example is reported in Fig. 4 for $K_{1}=0.1, \gamma_{1}=\gamma_{2}=0.05$, and $G=1.28$, and different $K_{2}$ values from 0 to 0.5 . In this case $G_{\mathrm{p}}=1.3$ (see Fig. 6).

The overall losses of the compound device control the fullwidth at half-maximum (FWHM) and the amplitude of the maximum. As an example, considering a specific design with $K_{1}$ and $K_{2}$ values making $\phi_{\mathrm{s}} \phi_{z} \sim 0$, the amplitude of the maximum output power in (29) is given by

$$
\left|\frac{E_{3}}{E_{1}}\right|_{\max }^{2}=\left(\frac{\sqrt{G_{\mathrm{c}}}-\sqrt{G}}{\sqrt{G_{\mathrm{p}}}-\sqrt{G}}\right)^{2}\left(\frac{G_{\mathrm{p}}}{G_{\mathrm{c}}}\right)^{2} .
$$

On the other hand, the FWHM bandwidth of the selective filter $\Delta f$ is obtained by finding the detuning where the maximum power in (32) decreases to half its value [5]. In deriving the FWHM, the output power is used. This is given by (29) and the approximation near resonances. In the high finesse case, $\cos (\theta) \sim 1-1 / 2 \theta^{2}$. By assuming $K_{1}$ and $K_{2}$ values, this relationship becomes $\phi_{\mathrm{s}} \sim \phi_{z} \sim 0$. This procedure yields

$$
\Delta f=\frac{\mathrm{FSR}}{\pi} \frac{\left(1-D_{0}\right)}{\sqrt{D_{0}}}
$$

where we can see that the FWHM is independent of $K_{2}$.

The finesse can be obtained in a straightforward manner from the previous expression.

\section{B. Designing a Notch Filter}

On the other hand, in designing a notch filter, the ratio $G_{\mathrm{c}} / G$ must be close to 1 . Tuning can be achieved through the coupling coefficients $K_{1}$ and $K_{2}$, but $K_{2}$ values must be within the range imposed by (24) for a specific $K_{1}$ value. In this case, the device will always be stable. An example can be seen in Fig. 5, with $K_{1}=0.4, \gamma_{1}=\gamma_{2}=0.05$, and $G=1.13$, and with different $K_{2}$ values from 0 to 0.5 . In this case, $G_{\mathrm{p}}=1.8>G$.

In our equations, we have considered the losses and a possible gain for compensating the losses or for giving more 


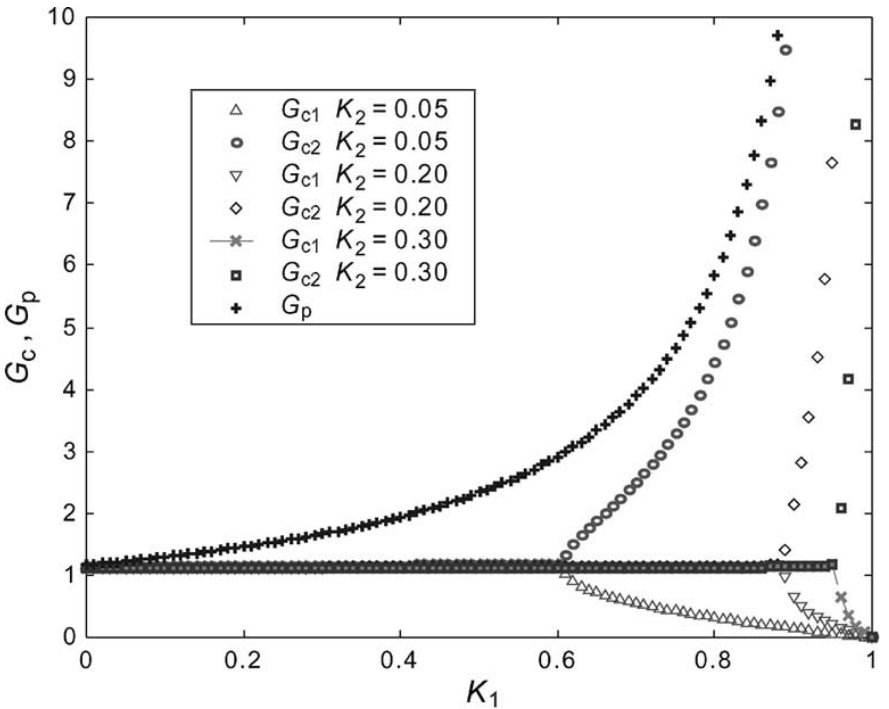

Fig. 6. Pole and zero gains, $G_{\mathrm{p}}$ and $G_{\mathrm{C}}$, versus $K_{1}$ with $K_{2}$ as a parameter. $\gamma_{1}=\gamma_{2}=0.05$, of the normalized transfer function at port $E_{3}$, for the active RR with a Sagnac loop.

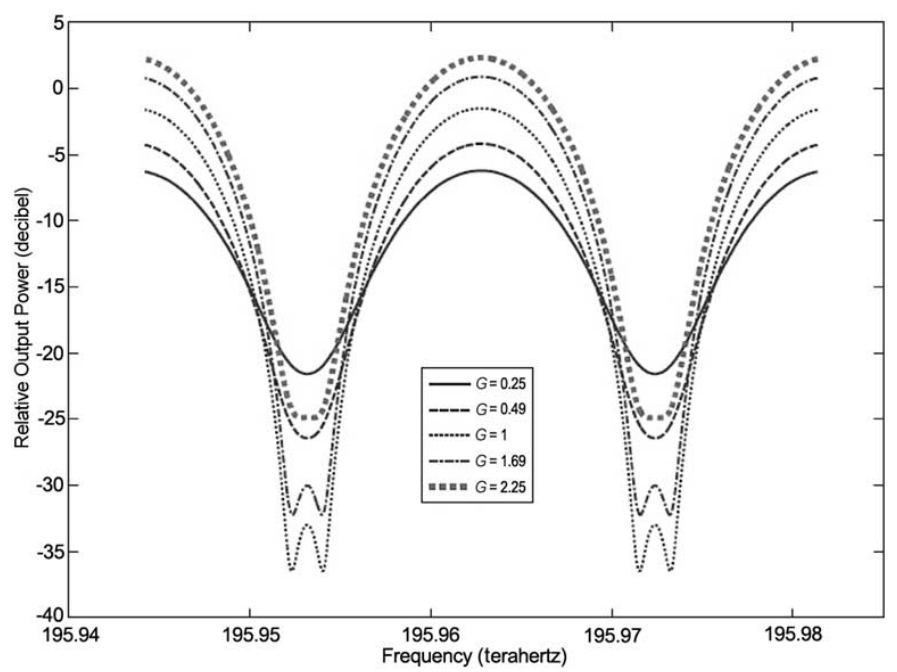

Fig. 7. Output power versus frequency of the RR with a Sagnac Loop: $K_{1}=0.92, K_{2}=0.75$ and, $G$ in legend represents total loss or gain effect so including $\mathrm{e}^{-2 \alpha L_{T}}, \gamma_{1}, \gamma_{2}$, and power gain.

flexibility to the designs. The effect of losses is very relevant; it has been shown that, depending on the relation between $G$, $G_{\mathrm{c}}$, and $G_{\mathrm{p}}$, it is possible to have a selective or notch filter. FWHM and minimum/maximum amplitude are also dependent on losses. Even resonance frequencies can be affected by this parameter, if having great losses. This effect can be seen in the example shown in Fig. 7, where the output power versus frequency for $K_{1}=0.92, \gamma_{1}=\gamma_{2}=0.075, K_{2}=0.75$, and different losses, are plotted. It can be seen that the two minima are quite close, so they can be distinguished only if gains close to $G_{\mathrm{c}}$ are used. In this case, from (26), a $G_{\mathrm{c}}=1.26$ is obtained, so the minima going away from this value are not shown.

\section{Dispersion Properties}

Potential degrading effects of a filter cascade are the dispersion problems caused by nonlinear filter phase responses
[2], [38]. On the other hand, these filters are used in some cases as dispersion compensators [4]. In any case, it is very important to know the dispersion properties of the filter. For the specific conditions reported in (24), the output power is given by (29) and in the $z$-transform domain, the transfer function can be expressed as

$$
\frac{E_{3}}{E_{1}}=K_{0} z^{-2} \prod_{i=1}^{2}\left[\frac{\left(z-Z c_{i}\right)}{\left(1-Z p_{i} z^{-1}\right)}\right]
$$

where $K_{0}$ is a constant.

The differentiation of the transfer function phase with respect to $\omega$ gives the group-delay time of the filter, using (29) and (34), under the conditions imposed by (24), the following expression is obtained:

$$
\begin{aligned}
\tau=\tau_{0} & \sum_{i=1}^{2} r_{\mathrm{c} i} \frac{\cos \left(-\beta L_{\mathrm{T}}+(-1)^{i} \phi_{z}\right)-r_{\mathrm{c} i}}{1-2 r_{\mathrm{c} i} \cos \left(-\beta L_{\mathrm{T}}+(-1)^{i} \phi_{z}\right)+r_{\mathrm{c} i}^{2}} \\
& -\sum_{i=1}^{2} r_{\mathrm{p} i} \frac{\cos \left(-\beta L_{\mathrm{T}}+(-1)^{i} \phi_{\mathrm{s}}\right)-r_{\mathrm{p} i}}{1-2 r_{\mathrm{p} i} \cos \left(-\beta L_{\mathrm{T}}+(-1)^{i} \phi_{\mathrm{s}}\right)+r_{\mathrm{p} i}^{2}}
\end{aligned}
$$

with

$$
r_{\mathrm{c} i}=\sqrt{\frac{G}{G_{\mathrm{c}}}} ; \quad r_{\mathrm{p} i}=\sqrt{\frac{G}{G_{\mathrm{p}}}} \quad \forall i ; \quad \tau_{0}=\frac{1}{\mathrm{FSR}}
$$

Dispersion measures the rate of change of the group-delay with respect to wavelength. The per-unit length dispersion is defined as

$$
D=L_{\mathrm{T}}^{-1} \frac{\partial \tau}{\partial \lambda}=-\frac{2 \pi c}{\lambda^{2} L_{\mathrm{T}}^{2}} \tau_{o} \frac{\partial \tau}{\partial \beta}
$$

which can be calculated from (35).

A proper selection of the location of the poles and zeros allows for the control of filter dispersion.

In Fig. 8, the group-delay time for the selective filter previously designed is shown, the tuning of which is shown in Fig. 4. In Fig. 8(a), the group-delay time is shown as a function of frequency and $K_{2}$ at a fixed value of $K_{1}, G$, and losses. The fixed values are the same as those for Fig. 4. The process of how the group-delay, and consequently, the dispersion, is tuned is clearly visible in Fig. 8(b), where the group-delay time of Fig. 8(a) is shown for five specific $K_{2}$ values: $K_{2}=0$, $K_{2}=0.25, K_{2}=0.50, K_{2}=0.75$, and $K_{2}=1$.

The group-delay time for the notch filter previously designed is shown in Fig. 9. It is plotted as a function of frequency, at a fixed value of $K_{1}, G$, and losses, and the parameter is $K_{2}$. The fixed and the $K_{2}$ parameter values are the same as those for Fig. 5.

In Fig. 10, the group-delay time of a device as a function of frequency is plotted. The device has a positive and negative value and sharp and smooth responses, depending on the parameter $K_{2}$. The fixed values are $K_{1}=0.9$, and overall power losses of $0.25\left(G=0.25, \gamma_{1}=\gamma_{2}=0=\alpha\right)$. 


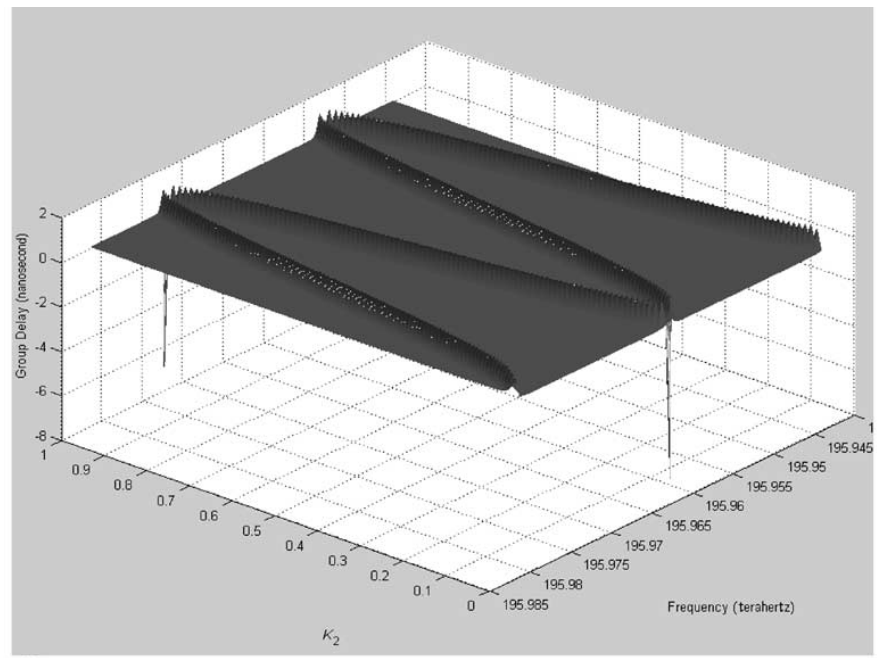

(a)

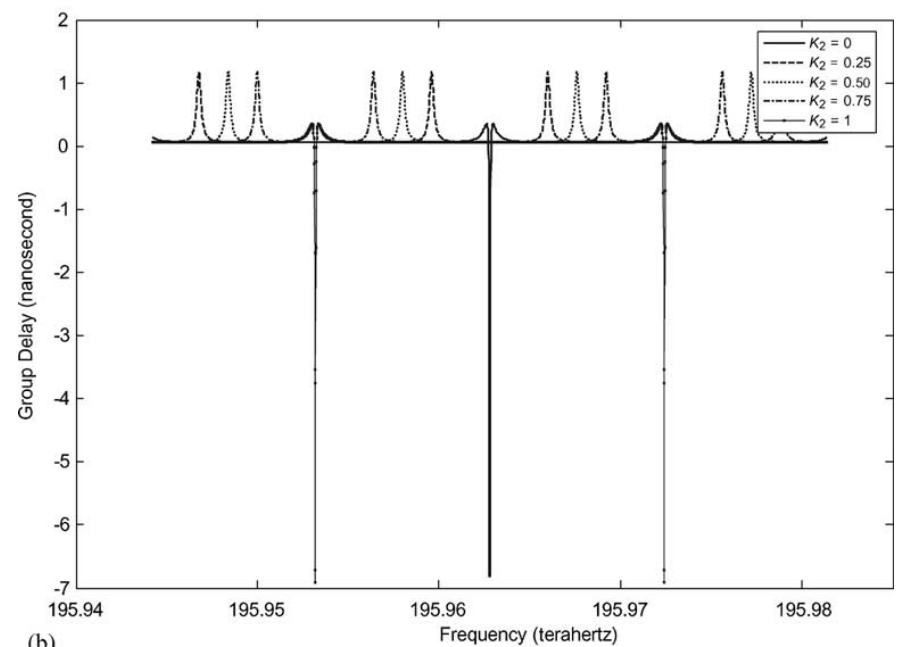

(b)

Frequency (terahertz)

Fig. 8. (a) Group-delay time of the active RR with a Sagnac loop as a function of frequency and $K_{2}$ at a fixed value of $K_{1}, G$, and losses. The fixed values are the same as those for Fig. 4. (b) Group-delay time of (a) for five specific $K_{2}$ values: $0,0.25,0.50,0.75$, and 1 .

\section{Coupling-Ratio Tolerance Effect on Tuning}

The study of tolerance is very important because it helps us estimate the specific tolerance of implemented devices, depending on the basic elements that they are made of. A study of parameter variation influence on design specifications for an RR-based device is reported in [7]; fabrication tolerances and temperature effects on design specifications and on working wavelength are taken into account, and some specific values using fiber technology are included. But nothing is said about coupling-ratio accuracy on wavelength tuning, which is going to be described here for the RR with a Sagnac loop configuration.

The tolerance study is usually made around a working design point, which will be defined by the subscript $q$. The resonant frequency location, obtained from (18) in a selective filter and from (27) in a notch filter, depends on temperature through $L_{\mathrm{T}}$, and $n_{\mathrm{ef}}$ as in any RR, but it also depends on $K_{2}$ and $K_{1}$. The accuracy on these coupling coefficients determines the resonant frequency location accuracy, and this dependence is

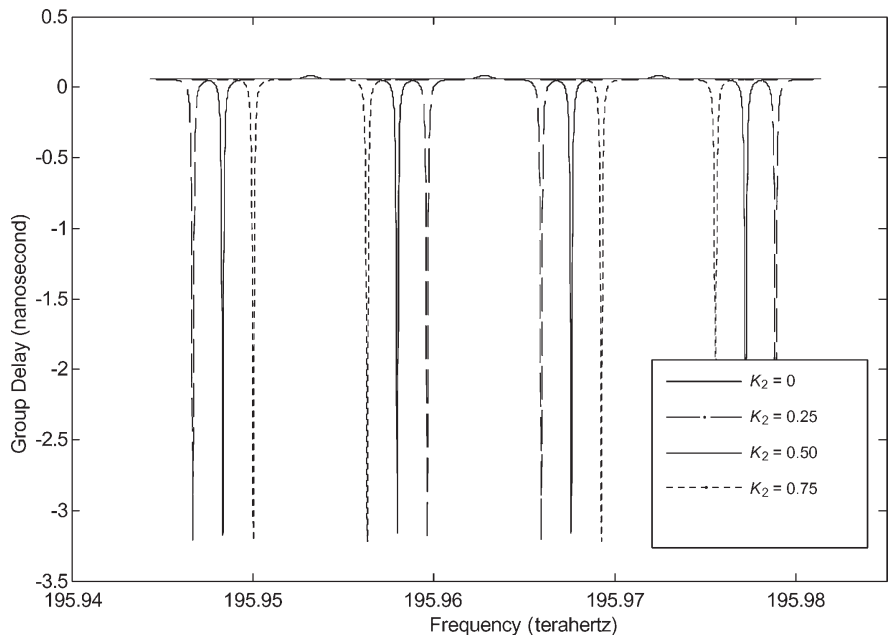

Fig. 9. Group-delay time of an RR with a Sagnac loop as a function of frequency, at a fixed value of $K_{1}, G$, and losses, and the parameter is $K_{2}$. The fixed and the $K_{2}$ parameter values are the same as those for Fig. 5.

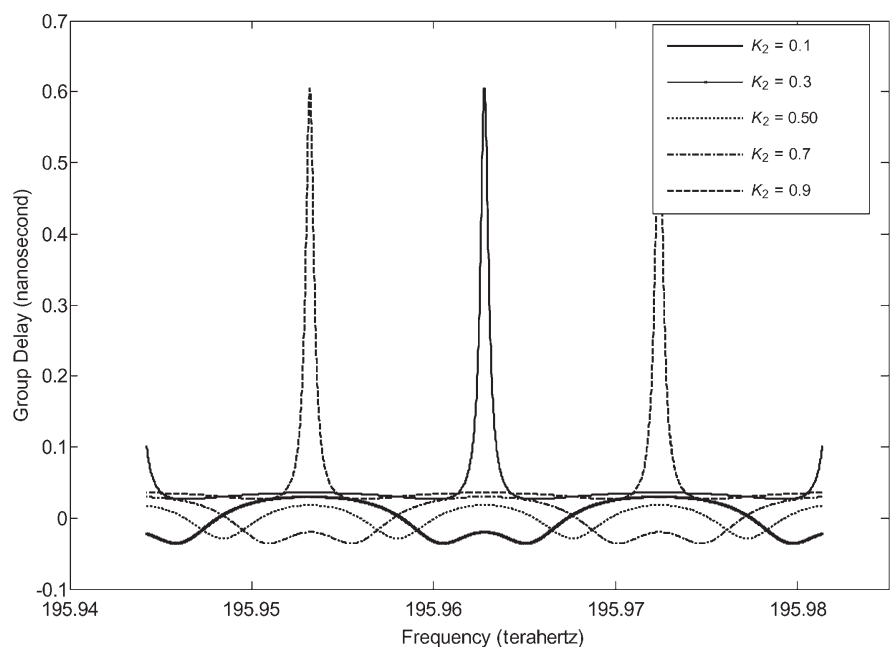

Fig. 10. Group-delay time (picosecond) of the RR with a Sagnac loop as a function of frequency: $K_{1}=0.9, G=0.25, \gamma_{1}=\gamma_{2}=\alpha=0$, and with $K_{2}$ as a parameter.

given by

$$
\left|\Delta f_{1,2}\right|=\left(\frac{\partial f_{1,2}}{\partial K_{2}}\right) \Delta K_{2}+\left(\frac{\partial f_{1,2}}{\partial K_{1}}\right) \Delta K_{1} .
$$

For the selective filter, from (16) and (18), it can be seen that only the first term is not equal to 0 and the resonance frequency accuracy is given by

$$
\left|\Delta f_{1,2}\right|=\frac{\mathrm{FSR}}{2 \pi}\left(\frac{\partial \phi_{\mathrm{s}}}{\partial K_{2}}\right) \Delta K_{2}
$$

with

$$
\left|\frac{\partial \phi_{\mathrm{s}}}{\partial K_{2}}\right|=\left(\frac{1}{\left(1-2 K_{2}\right)^{2} \sqrt{K_{2}\left(1-K_{2}\right)}}\right) .
$$

The previously mentioned relationship becomes more critical if $K_{2}$ is close to $0.5,0$, and 1 . For example, for $K_{2}=$ 0.49 and $\Delta K_{2}=0.001$, relative deviation $\left(1 / \phi_{\mathrm{s} q} \times \Delta K_{2} \times\right.$ 
$\left.\left[\delta \phi_{\mathrm{S}} / \delta K_{2}\right]_{q} \times 100\right)$ is less than $10 \%$, while for $K_{2}=0.3$ it is less than $0.6 \%$.

For the notch filter, from (27) and (28), it can be seen that both terms of (38) are now not equal to 0 , and the resonance frequency accuracy is given by

$$
\left|\Delta f_{1,2}\right|=\frac{\mathrm{FSR}}{2 \pi}\left[\left(\frac{\partial \phi_{z}}{\partial K_{2}}\right) \Delta K_{2}+\left(\frac{\partial \phi_{z}}{\partial K_{1}}\right) \Delta K_{1}\right]
$$

with (42) and (43), shown at the bottom of the page.

In this case, the worst accuracy is again obtained for $K_{2}$ values close to 0.5 . On the other hand, one term is positive and the other is negative, so the accuracy is smaller than the bigger term. The dominant effect always comes from $K_{2}$ tolerances, which is clear if $K_{1}$ tends to 0 ; if $K_{1}$ tends towards 1 , and (24) must be fulfilled so that this happens for $K_{2}$, values close to 0.5 and the denominator of (43) have a square term in $\left(1-2 \mathrm{~K}_{2}\right)$, so again, it is dominant. For example, for $K_{2}=$ $K_{1}=0.49$ and $\Delta K_{2}=\Delta K_{1}=\Delta K=0.001$, relative deviation $\left(1 / \phi_{z q} \times\left[\left(\delta \phi_{z} / \delta K_{2}+\delta \phi_{z} / \delta K_{1}\right) \times \Delta K\right]_{q} \times 100\right)$ is less than $10 \%$, while for $K_{2}=0.3$, it is less than $0.59 \%$.

\section{CONCLUSION}

Novel filter architecture based on co- and counterpropagation signals are presented. Equations describing the output field frequency response for the different design parameters are reported. The same expressions are derived using two different techniques, the matrix formalism and the $z$-transform technique. They are particularized for a specific architecture made of a Sagnac loop in an RR. The multireflections originated by the loop are controlled through the gain or losses and through a coupling coefficient that is responsible for the tuning. Simple equations for designing and tuning are reported, along with rules for designing tunable selective or notch filters. The effect of losses (waveguide and coupler losses and optionally optical gain), the filter dispersion properties, and the coupler coefficient tolerances on resonant frequency accuracy are also discussed.

The compound device analyzed has some physical characteristics similar to the RR ones that can be exploited in photonic applications, which are complemented with the property of being tunable with the coupling coefficient under specific design conditions. These properties are its notch filter amplitude characteristics near critical coupling, and the magnification of the signal amplitude circulating in the ring, which is associated with energy storage. The last one can be used in designing laser oscillators. Using the standard method of photonic filter synthesis, the compound device can be used for designing frequency filters of different orders and dispersion compensation filters.

Previous measurements on passive notch filter in fiberoptic technology validate the expressions derived and the novel tuning principle. The structure can be developed using integrated optics technologies.

\section{APPENDIX A}

\section{COMPOUnd RR Using THE MATRIX Formalisms}

The filter architecture is shown in Fig. 1. The transfermatrix method is used for calculating the transfer function of a compound device that is made of concatenated elementary devices; in our filter, the decomposition used is shown in Fig. 2. In the following, we are going to describe the expressions of the different matrices involved.

The coupler transmission matrix is given by

$$
\begin{aligned}
{[a c] } & =\left[\begin{array}{cc}
\frac{1}{t_{a c}} & -\frac{r_{a c}}{t_{a c}} \\
\frac{r_{a c}}{t_{a c}} & \frac{t_{a c}^{2}-r_{a c}^{2}}{t_{a c}}
\end{array}\right] \\
t_{a c} & =j\left[\left(1-\gamma_{1}\right) K_{1}\right]^{\frac{1}{2}} \\
r_{a c} & =\left[\left(1-\gamma_{1}\right)\left(1-K_{1}\right)\right]^{\frac{1}{2}}
\end{aligned}
$$

where $K_{1}$ and $\gamma_{1}$ are the coupling coefficient and the excess loss of the input coupler, respectively.

The transfer matrix of the waveguides and the transmission functions within them are given by

$$
[g]=\left[\begin{array}{ll}
\Pi T_{x_{i}} \mathrm{e}^{(\alpha+j \beta) l_{a}} & 0 \\
0 & \Pi T_{y_{i}} \mathrm{e}^{-(\alpha+j \beta)\left(L_{\mathrm{T}}-l_{a}\right)}
\end{array}\right]
$$

where $\alpha$ and $\beta$ are the waveguide attenuation and the propagation constant, respectively, $L_{\mathrm{T}}$ is the total waveguide length, $l_{a}$ is the waveguide length connecting the input coupler and the TRF (see Fig. 1) and $\Pi_{1}^{N}\left(T_{x_{i}}\right)$ and $\Pi_{1}^{M}\left(T_{y_{i}}\right)$ are the $N$ transmission transfer functions between the input coupler and the TRF and the $M$ transmission transfer functions between the TRF and the input coupler, respectively.

The TRF transfer matrix is given by

$$
[\mathrm{TRF}]=\left[\begin{array}{cc}
\frac{1}{\mathrm{FR}} & -\frac{\mathrm{FT}}{\mathrm{FR}} \\
\frac{\mathrm{FT}}{\mathrm{FR}} & \frac{\mathrm{FR}^{2}-\mathrm{FT}^{2}}{2}
\end{array}\right]
$$

$$
\left|\frac{\partial \phi_{z}}{\partial K_{2}}\right|=\left(\frac{8\left(1-K_{1}\right)}{\left(1-2 K_{2}\right)^{2}\left(2-K_{1}\right) \sqrt{-K_{1}^{2}\left(1-2 K_{2}\right)^{2}+16\left(1-K_{1}\right)\left(1-K_{2}\right) K_{2}}}\right)
$$

$$
\left|\frac{\partial \phi_{z}}{\partial K_{1}}\right|=\left(\frac{-2 K_{1}}{\left(1-2 K_{2}\right)\left(2-K_{1}\right)^{2} \sqrt{-K_{1}^{2}\left(1-2 K_{2}\right)^{2}+16\left(1-K_{1}\right)\left(1-K_{2}\right) K_{2}}}\right)
$$


It represents a general TRF, and not only a symmetric discontinuity as in [13].

The transfer matrices of the original elements and their mirror images are equals $[g]=\left[g^{\prime}\right]$ and $[a c]=\left[a c^{\prime}\right]$.

\section{APPENDIX B}

\section{COMPOUND RR USING THE $z$-TRANSFORM TECHNIQUE}

The filter architecture is shown in Fig. 1. In deriving the transfer function in $z$ at output port $E_{3}$, we use the following equations, considering the TRF placed at the middle of the ring:

$$
\begin{aligned}
& E_{3}=\left(1-\gamma_{1}\right)^{\frac{1}{2}}\left[\left(1-K_{1}\right)^{\frac{1}{2}} E_{1}+j K_{1}^{\frac{1}{2}} E_{2}\right] \\
& E_{4}=\left(1-\gamma_{1}\right)^{\frac{1}{2}}\left[j K_{1}^{\frac{1}{2}} E_{1}+\left(1-K_{1}\right)^{\frac{1}{2}} E_{2}\right] \\
& E_{2}=\prod_{i=1}^{M} T_{y_{i}} \cdot z^{-1}\left[\prod_{i=1}^{N} T_{x_{i}} \cdot \mathrm{FT} \cdot E_{4}+\prod_{i=1}^{M} T_{y_{i}} \cdot \mathrm{FR} \cdot E_{2 b}\right]
\end{aligned}
$$

$$
\begin{aligned}
& E_{2 b}=\left(1-\gamma_{1}\right)^{\frac{1}{2}}\left(1-K_{1}\right)^{\frac{1}{2}} E_{4 b} \\
& E_{4 b}=\prod_{i=1}^{N} T_{x_{i}} \cdot z^{-1}\left[\prod_{i=1}^{M} T_{y_{i}} \cdot \mathrm{FT} \cdot E_{2 b}+\prod_{i=1}^{N} T_{x_{i}} \cdot \mathrm{FR} \cdot E_{4}\right]
\end{aligned}
$$

where FR and FT are the transfer functions in reflection and in transmission, respectively, of the TRF, and $z^{-1}$ represents the unit delay related to $L_{\mathrm{T}}$.

Combining the previous equations, the transfer function at port $E_{3}$ reported in (5) is obtained.

\section{ACKNOWLEDGMENT}

The authors would like to thank Prof. O. Schwelb for fruitful comments.

\section{REFERENCES}

[1] G. Rossi, O. Jerphagnon, B. Olsson, and D. J. Blumenthal, "Optical SCM data extraction using a fiber-loop mirror for WDM network systems," IEEE Photon. Technol. Lett., vol. 12, no. 7, pp. 897-899, Jul. 2000.

[2] G. Lenz, B. J. Eggleton, C. R. Giles, C. K. Madsen, and R. E. Slusher, "Dispersive properties of optical filters for WDM systems," IEEE J. Quantum Electron., vol. 34, no. 8, pp. 1390-1402, Aug. 1998.

[3] D. Sadot and E. Boimovich, "Tunable optical filters for dense WDM networks," IEEE Commun. Mag., vol. 36, no. 12, pp. 50-55, Dec. 1998.

[4] C. K. Madsen and J. H. Zhao, Optical Filter Design and Analysis, A Signal Processing Approach. New York: Wiley, 1999.

[5] O. Schwelb, "Transmission, group delay, and dispersion in single-ring optical resonators and add/drop filters-a tutorial overview," J. Lightw. Technol., vol. 22, no. 5, pp. 1380-1394, May 2004.

[6] N. Ngo and L. N. Binh, "Novel realization of monotonic butterworth-type lowpasss, highpass, and bandpass optical filters using phase-modulated fiber-optic interferometers and ring resonators," J. Lightw. Technol., vol. 12, no. 5, pp. 827-840, May 1994.

[7] C. Vázquez, S. Vargas, and J. M. S. Pena, "Design and tolerance analysis of a router using an amplified ring resonator and Bragg gratings," Appl. Opt., vol. 39, no. 12, pp. 1934-1940, 2000.

[8] R. Grover, V. Van, T. A. Ibrahim, P. P. Absil, L. C. Calhoun, F. G. Johnson, J. V. Hryniewicz, and P.-T. Ho, "Parallel-cascaded semiconductor microring resonators for high-order and wide-FSR filters," J. Lightw. Technol., vol. 20, no. 5, pp. 900-905, May 2002.
[9] S. A. Havstad, B. Fischer, A. E. Willner, and M. G. Wickham, "Loopmirror filters based on saturable-gain or -absorber gratings," Opt. Lett., vol. 24, no. 21, pp. 1466-1468, 1999.

[10] J. E. Heebner, P. Chak, S. Pereira, J. E. Sipe, and R. W. Boyd, "Distributed and localized feedback in microresonator sequences for linear and nonlinear optics," J. Opt. Soc. Amer., B, Opt. Phys., vol. 21, no. 10, pp. 18181832, 2004

[11] B. E. Little et al., "Very high-order microring resonator filters for WDM applications," IEEE Photon. Technol. Lett., vol. 16, no. 10, pp. 22632265, Oct. 2004.

[12] C. Vázquez, F. Hernández-Gil, and M. López-Amo, "Tunable ring resonator filter for OFDM transmission systems," Microw. Opt. Technol. Lett., vol. 8, no. 6, pp. 321-323, Apr. 1995.

[13] R. W. Boyd and J. E. Heebner, "Sensitive disk resonator photonic biosensor," Appl. Opt., vol. 40, no. 31, pp. 5742-5747, 2001.

[14] J. E. Heebner and R. W. Boyd, "Enhanced all-optical switching by use of a nonlinear fiber ring resonator," Opt. Lett., vol. 24 , no. 12, pp. 847-849, 1999.

[15] P. Urquhart, "Compound optical-fiber-based resonators," J. Opt. Soc. Amer. A, vol. 5, no. 6, pp. 803-812, 1988.

[16] J. Zhang and J. W. Y. Lit, "Compound fiber ring resonator: theory," J. Opt. Soc. Amer. A, vol. 11, no. 6, pp. 1867-1873, 1994.

[17] _ _ "All fiber compound ring resonator with a ring filter," J. Lightw. Technol., vol. 12, no. 7, pp. 1256-1262, Dec. 1994.

[18] O. Schwelb and I. Frigyes, "All-optical tunable filters built with discontinuity-assisted ring resonators," J. Lightw. Technol., vol. 19, no. 3, pp. 384-386, Mar. 2001

[19] S. Vargas, C. Vázquez, and J. M. S. Pena, "Wide tunable filters using a recirculating delay line with multi-reflections," Opt. Eng., vol. 41, no. 5, pp. 926-927, 2002.

[20] — "Novel tunable optical filter employing a fiber loop mirror for synthesis applications in WDM," in 14th Annu. Meeting IEEE Lasers and Electro-Optics Society, San Diego, CA, 2001, vol. II, pp. 899-900.

[21] C. Vázquez, S. Vargas, J. M. S. Pena, and P. Corredera, "Tunable optical filters using compound ring resonators for DWDM," IEEE Photon. Technol., vol. 15, no. 8, pp. 1085-1087, Aug. 2003.

[22] V. M. Menon, W. Tong, F. Xia, C. Li, and S. R. Forrest, "Nonreciprocity of counterpropagating signals in a monolithically integrated Sagnac interferometer," Opt. Lett., vol. 29, no. 5, pp. 513-515, 2004.

[23] D. G. Rabus, M. Hamacher, U. Troppenz, and H. Heidrich, "High-Q channel-dropping filters using ring resonators with integrated SOAs," IEEE Photon. Technol. Lett., vol. 14, no. 10, pp. 1442-1444, Oct. 2002.

[24] V. M. Menon, W. Tong, and S. R. Forrest, "Control of quality factor and critical coupling in microring resonators through integration of a semiconductor optical amplifier," IEEE Photon. Technol., vol. 16, no. 5, pp. 1343-1345, May 2004.

[25] K. Oda, S. Suzuki, H. Takahashi, and H. Toba, "An optical FDM distribution experiment using a high finesse waveguide-type double ring resonator," IEEE Photon. Technol., vol. 6, no. 8, pp. 1031-1034, Aug. 1994.

[26] D. G. Rabus and M. Hamacher, "MMI-coupled ring resonators in GaInAsP-InP," IEEE Photon. Technol., vol. 13, no. 8, pp. 812-814, Aug. 2001

[27] A. Melloni, R. Costa, P. Monguzzi, and M. Martinelli, "Ring-resonator filters in silicon oxynitride technology for dense wavelength-division multiplexing systems," Opt. Lett., vol. 28, no. 17, pp. 1567-1569, 2003.

[28] D. K. Armani, T. J. Kippenberg, S. M. Spillane, and K. J. Vahala, "Ultrahigh-Q toroid microcavity on a chip," Nature, vol. 421, no. 6926, pp. 925928, 2003.

[29] K. Djordjev, S. Choi, S. Chou, and P. Dapkus, "Microdisk tunable resonant filters and switches," IEEE Photon. Technol. Lett., vol. 14, no. 6, pp. 828830, Jun. 2002.

[30] B. E. Little et al., "Very high-order microring resonator filters for WDM applications," IEEE Photon. Technol. Lett., vol. 16, no. 10, pp. 22632265 , Oct. 2004.

[31] K. Djordjev, S. Choi, S. Chou, and P. Dapkus, "Vertically coupled InP microdisk switching devices with electroabsorptive active regions," IEEE Photon. Technol. Lett., vol. 14, no. 8, pp. 1115-1117, Aug. 2002.

[32] M. M. Lee and M. C. Wu, "A MEMS-actuated tunable microdisk resonator," in IEEE Int. Conf. Optical Microelectromechanical Systems (MEMS), Waikoloa, HI, 2003, pp. 28-29, MC3.

[33] W. M. J. Green, R. K. Lee, A. Yariv, and M. A. Scherer, "Control of optical waveguide-resonator coupling: Application to low-power optical modulation and switching," in IEEE Lasers and Electro-Optics Society (LEOS) Annu. Meeting Conf. Proc., Tucson, AZ, 2003, vol. 1, pp. 130131. MM3. 
[34] W. M. J. Green, R. K. Lee, G. A. DeRose, A. Scherer, and A. Yariv, "Hybrid InGaAsP-InP Mach-Zehnder racetrack resonator for thermooptic switching and coupling control," Opt. Express, vol. 13, no. 5, pp. 16511659, 2005.

[35] R. H. Qu, H. Zhao, Z. J. Fang, E. Marin, and J. P. Meunier, "Configurable wavelength-selective switch based on fiber grating and fiber loop mirror," IEEE Photon. Technol., vol. 12, no. 10, pp. 1343-1345, Oct. 2000.

[36] X. Shu, S. Jiang, and D. Huang, "Fiber grating Sagnac loop and its multiwavelegth-laser," IEEE Photon. Technol., vol. 12, no. 8, pp. 980982, Aug. 2000.

[37] I. Golub and E. Simova, "Ring resonator in a Sagnac interferometer as a birefringence magnifier," Opt. Lett., vol. 30, no. 1, pp. 87-89, 2005.

[38] J. D. Downie and A. B. Ruffin, "Analysis of signal distortion and crosstalk penalties induced by optical filters in optical networks," J. Lightw. Technol., vol. 21, no. 9, pp. 1876-1886, Sep. 2003.

Carmen Vázquez (M’99) was born in Madrid, Spain, on May 1968. She received the M.S. degree in physics (electronics) from Complutense University of Madrid, Madrid, Spain, in 1991 and the Ph.D. degree from the Telecommunications Engineering School, Polytechnic University of Madrid (UPM), Madrid, Spain, in 1995.

She enjoyed a fellowship at TELECOM, Denmark, in 1991, working on erbium-doped fiber amplifiers. From 1992 to October 1995, she worked at the Optoelectronics Division of Telefónica Investigación y Desarrollo, Madrid, Spain. She was involved in III-V integrated optics devices characterization, design, and fabrication. In October 1995, she joined University Carlos III of Madrid, Madrid, Spain, where she is currently working as an Associate Professor and Head of the Electronics Technology Department. Her current work includes the design of fiber optics, liquid crystals (LC), and integrated optic devices, and their application to optical communications, instrumentation, and sensor networks.

Dr. Vázquez is a Member of The International Society for Optical Engineers (SPIE), Optical Society of America (OSA), and Sociedad Española de Óptica (SEDO).
Salvador Elías Vargas was born in Panamá, in 1973. He received the M.S. degree in electrical and electronic engineering from Universidad Tecnológica de Panamá, Panama, in 1996, and the Ph.D. degree at University Carlos III of Madrid, Madrid, Spain, in December 2003.

He was an Assistant Professor in Universidad Tecnológica de Panamá, Panama, from January to September of 1997. In October 1997, he enjoyed a fellowship from the Spanish International Cooperation Agency to do studies in Carlos III University of Madrid, Madrid, Spain. He is currently an Assistant Professor in the Electronics Department of Carlos III University of Madrid, Madrid, Spain. His main research interests include synthesis of photonic filters, tunable photonic filters, liquid crystals (LC), and polarization devices, and their applications to optical communications.

José Manuel Sánchez Pena (A'95-SM'03) received the M.S. and Ph.D degrees in telecommunication engineering from the Polytechnic University of Madrid, Madrid, Spain, in 1988 and 1993, respectively.

He was a Visiting Researcher at Exeter University, Devon, U.K., from February to September of 1993. In March 1995, he joined University Carlos III of Madrid, Madrid, Spain, where he is currently an Associate Professor and Head of the Displays and Photonic Applications Group of the Electronic Technology Department. His main research interests include electrooptical characterization of liquid-crystal displays, design of optical sensors and optoelectronic instrumentation for rehabilitation technology applications, and optical communications.

Dr. Pena is a Member of The International Society for Optical Engineers (SPIE), International Liquid Crystal Society (ILCS), and International Society for Augmentative \& Alternative Communication (ISAAC). 